\title{
EchoGéo
}

\section{Les enjeux de la grande vitesse ferroviaire dans l'émergence de coopérations interubaines}

La perspective du corridor de développement pour l'espace RhinRhône?

The stakes of high speed train lines rail in the birth of intercity cooperations: the development corridor prospect for Rhin-Rhône space?

\section{Guillaume Carrouet}

\section{OpenEdition}

\section{Journals}

\section{Édition électronique}

URL : https://journals.openedition.org/echogeo/17970

DOI : $10.4000 /$ echogeo. 17970

ISSN : 1963-1197

\section{Éditeur}

Pôle de recherche pour l'organisation et la diffusion de l'information géographique (CNRS UMR 8586)

\section{Référence électronique}

Guillaume Carrouet, « Les enjeux de la grande vitesse ferroviaire dans l'émergence de coopérations interubaines », EchoGéo [En ligne], 49 | 2019, mis en ligne le 25 octobre 2019, consulté le 11 août 2021 URL : http://journals.openedition.org/echogeo/17970 ; DOI : https://doi.org/10.4000/echogeo.17970

Ce document a été généré automatiquement le 11 août 2021.

EchoGéo est mis à disposition selon les termes de la licence Creative Commons Attribution - Pas d'Utilisation Commerciale - Pas de Modification 4.0 International (CC BY-NC-ND) 


\section{Les enjeux de la grande vitesse ferroviaire dans l'émergence de coopérations interubaines}

La perspective du corridor de développement pour l'espace RhinRhône?

The stakes of high speed train lines rail in the birth of intercity cooperations: the development corridor prospect for Rhin-Rhône space?

\section{Guillaume Carrouet}

\section{Introduction}

1 Dans la littérature scientifique, la portion d'espace qui s'étend de Dijon à Bâle a fait l'objet d'un intérêt particulier de la part des géographes. Deux travaux de doctorat (Gibert, 1930 ; Dézert, 1969), portant sur la partie nord de l'ancienne région FrancheComté, et une thèse plus récente (Wœssner, 1996), au périmètre correspondant globalement à la première délimitation, se sont centrés sur la thématique du développement industriel. Ils ont tous mis en évidence son intérêt pour les circulations en France et à l'échelle de l'isthme européen. L'espace Rhin-Rhône semble donc jouer le rôle d'interface car il représente une zone de contact entre deux systèmes distincts, où des phénomènes originaux peuvent se produire (De Ruffray, 2004). Plus récemment, l'arrivée de la grande vitesse ferroviaire via la mise en service de la branche Est de la LGV Rhin-Rhône, en plus de conforter sa dimension circulatoire, offre des perspectives de recherche dans le champ plus vaste des interactions entre transports et territoires.

Cette contribution discute de l'impact d'un grand équipement de transport sur un espace dépourvu de dimension territoriale dans le sens où aucun périmètre institutionnel n'intègre l'ensemble des villes. Pourtant, les acteurs locaux ont vu dans les gains d'accessibilité un facteur favorable au développement de coopérations institutionnelles à travers la création de la Métropole Rhin-Rhône, un réseau de villes 
correspondant approximativement à la portion Dijon-Bâle, qui peut être identifié comme une première réalité territoriale. Sans toutefois revenir en détail sur le mythe persistant des effets structurants chez les décideurs locaux, l'objectif est d'analyser un cas précis où convergent des configurations physiques entre Rhin et Rhône favorables aux circulations, une LGV à la dimension régionale marquée et les attentes des acteurs locaux en matière de développement économique. En d'autres termes, dans quelle mesure la LGV est susceptible d'être à l'origine de coopérations interurbaines au sein de l'espace Rhin-Rhône? De fait, la question de l'appartenance de cet espace à la catégorie des corridors de développement, outre la présence d'une dimension circulatoire, peut être posée puisque le critère de la gouvernance dans le développement de ceux-ci semble primordial. Comme l'indique J. Debrie et C. Comtois (2010) : « la gouvernance d'un corridor dans un contexte de croissance des activités repose sur une forme de coordination entre chaque composante territoriale et économique ». Les configurations territoriales et retistiques invitent à questionner la perspective du corridor de développement pour l'espace Rhin-Rhône.

3 L'analyse se base sur une méthodologie qualitative mise en œuvre dans une thèse de doctorat, avec des entretiens semi-directifs centrés sur les attentes des acteurs locaux. Ces interviews ont été menées quelques mois avant la mise en service de la LGV. Pour pallier le manque de recul temporel nécessaire à l'analyse de processus pouvant apparaître à moyen ou à long terme, le choix a été de considérer l'appropriation des acteurs locaux. Cette entrée permet « d'appréhender les acteurs et leurs pratiques, les rapports et les processus sociaux plutôt que des "objets géographiques" trop souvent figés» (Ripoll et Veschambre, 2005). Dans le contexte présent, l'entrée par l'appropriation des acteurs locaux de la grande vitesse ferroviaire, saisie grâce aux entretiens, permet d'identifier les représentations que ces derniers se font de cette infrastructure et les projections des éventuellement transformations induites sur leur territoire, y compris en matière de coopérations interurbaines et de développement local. Ce dispositif est complété par le recensement des projets de développement de valorisation aux abords des gares, en particulier en centre-ville, avec un suivi de huit années après la mise en service de l'infrastructure afin d'évaluer les effets de cette dernière sur les territoires desservis. L'émergence de projets de développement territoriaux aux abords des points de desserte espacés seulement d'une centaine de kilomètres ou 30 minutes en TGV est un repère pour l'identification de coopérations, socle du réseau de villes de la Métropole Rhin-Rhône. Concrètement, les coopérations pourraient s'incarner à travers l'existence d'un espace de projets au sein duquel les rivalités en matière de développement territorial s'effacent au profit d'un aménagement concerté. À l'inverse, une redondance des activités serait la preuve d'un effacement des coopérations au profit du développement local privilégié à proximité de chaque point de desserte.

4 Après avoir mobilisé la littérature sur les corridors de développement dans le but de discuter des conditions d'appartenance de l'espace Rhin-Rhône à cette catégorie, il est question de présenter l'originalité de la desserte de la branche Est et du réseau de villes créé dans la perspective de sa mise en service. La notion d'appropriation de cet équipement de transport a été choisie comme fil conducteur d'un troisième temps car elle reflète la capacité des acteurs à faire émerger collectivement des projets de développement territoriaux, ce qu'appelle de ses vœux la Métropole Rhin-Rhône. 


\section{L'espace Rhin-Rhône : un corridor imparfait}

5 Dans la littérature scientifique et les discours, l'espace Rhin-Rhône a régulièrement été identifié par le vocable corridor (Wœssner, 2006). Si cet espace est associé à de grands projets de transport, tels que le Canal à Grand Gabarit Rhin-Rhône ou le TGV éponyme, à certains égards, cette appellation est à questionner.

\section{Un couloir favorisant les circulations à l'échelle de l'isthme européen}

6 À la lecture de la carte de l'Europe, l'espace Rhin-Rhône offre une voie de passage naturelle entre les deux fleuves et constitue un maillon pour les échanges entre Mer du Nord et Mer Méditerranée. À un niveau plus fin, il s'étend du sud de l'Alsace jusqu'à la Plaine de Saône aux environs de Chalon-sur-Saône et comprend, dans sa partie centrale, la Trouée de Belfort et la vallée du Doubs (illustration 1). Ce couloir naturel déterminé par la géographie physique a favorisé l'implantation d'infrastructures de transport dont la vocation est de desservir les grands foyers urbains à proximité et la desserte des villes de taille moyenne qui le jalonnent. L'autoroute A36 mise en service dans le milieu des années 1980 est un grand équipement de transport dont l'objectif est de réduire les distances-temps entre plusieurs métropoles françaises et européennes. Cette fonction d'espace de connexion à l'échelle de l'isthme est également visible à travers les caractéristiques de la branche Est, premier tronçon du TGV Rhin-Rhône, mis en service en 2011. À l'image de cette première autoroute, elle a amélioré de manière significative les relations entre Strasbourg et Lyon, dernier côté du triangle ParisStrasbourg-Lyon dépourvu de grande vitesse ferroviaire avant cette date (Richer et Bérion, 2010). À travers les réalisations de grands projets de transport se dessine la vocation première de l'espace Rhin-Rhône de permettre les circulations à l'échelle du continent européen. La projection d'un Canal à grand gabarit dès les années 1960, équipement abandonné officiellement en 1997, souligne d'autant plus cette fonction circulatoire. Dans une perspective plus large, l'histoire de l'Europe permet d'identifier l'espace Rhin-Rhône comme un maillon au regard de considérations stratégiques et commerciales. Lors de l'occupation romaine (Ollivro, 2000), il a joué le rôle de maillon dans les relations nord-sud à cette échelle. Cette fonction est restée d'actualité puisqu'il est partie prenante du réseau de corridor actuel défini par l'Union Européenne TEN-T (Trans-European Transport Network). Enfin, il s'inscrit dans les relations est-ouest, en particulier dans le mouvement général de rééquilibrage à l'échelle européenne (Dézert, 2003). À l'échelle française, l'espace Rhin-Rhône se positionne dans ces deux dynamiques spatiales, à l'image du projet VFCEA (Voie Ferrée Centre Europe Atlantique), qui prévoit une amélioration des liaisons ferroviaires transversales de voyageurs et de fret. 


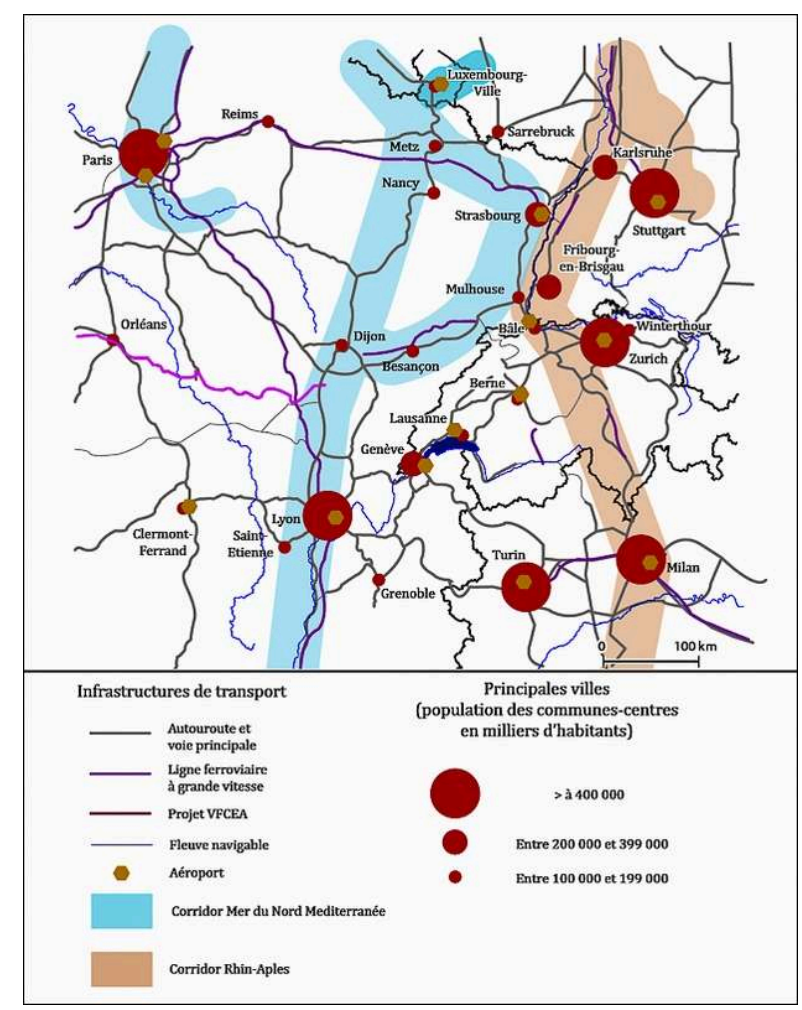

Source : Commission Européenne [site web] https://ec.europa.eu/transport/infrastructure/tentec/ tentec-portal/map/maps.html. Auteur : G. Carrouet, 2019.

Pourtant, le caractère stratégique d'interface est remis en question en raison de l'existence de plusieurs voies de passage remplissant les mêmes fonctions circulatoires. Par exemple, le projet de LGV Lyon-Turin fait figure de concurrent pour les relations ferroviaires entre Mer du Nord et Mer Méditerranée. Dans le même ordre d'idée, l'espace Rhin-Rhône, partie prenante du corridor Mer du Nord-Méditerranée appartenant au TEN-T, est concurrencé par le corridor Rhin-Alpes dont la fonction est de desservir les principaux ports (Northern range et façade Méditerranéenne) et la partie centrale de l'espace communautaire. Enfin, le maillon Rhin-Rhône n'est pas systématiquement choisi dans les projets d'amélioration de la desserte des réseaux de transport terrestres ferroviaires et fluviaux, les axes du Rhône à la Moselle jusqu'au Luxembourg, à l'ouest, la plaine d'Alsace par Strasbourg et Bâle, à l'est, favorisant également les circulations nord-sud.

8 À l'échelle française ${ }^{1}$, s'il est délicat de délimiter un espace dont l'existence ne s'inscrit pas dans des découpages institutionnels, ce maillon a pour caractéristique, à l'exception de Bâle, d'être composé d'un semi d'agglomérations de moyenne taille parmi lesquelles figurent Dijon, Besançon, Montbéliard, Belfort, Mulhouse et Fribourgen-Brisgau. Sa dimension transfrontalière accentue le morcellement de cet espace puisqu'il est composé de six départements français (Côte-d'Or, Jura, Doubs, HauteSaône, Territoire de Belfort et Haut-Rhin), trois cantons suisses (Jura, Bâle-Ville et Bâle campagne) et du district allemand de Fribourg-en-Brisgau appartenant au Land du Bade-Wurtemberg. 


\section{Le corridor Rhin-Rhône incarné dans sa dimension politique}

9 Le concept de corridor de développement semble manquer d'une définition précise (Arnold, 2005). Toutefois, la littérature scientifique dégage plusieurs caractéristiques qui permettent d'en décrire sa nature et sa fonction, dans un contexte où la notion de « corridor » Rhin-Rhône pose question.

En premier lieu, un corridor se définit par une concentration d'infrastructures de transport ou «bundles of infrastructures" (Priemus et Zonneveld, 2003), dont l'efficacité est primordiale (Comtois, 2012). Ces faisceaux accueillent des infrastructures destinées au transport de voyageurs et de fret. Le corridor est également décrit selon sa morphologie (Comtois, 2012). Il peut notamment revêtir la forme d'une chaîne avec « une séquence de corridors dont le terminal de sortie de l'un coïncide avec le terminal d'entrée de l'autre » (Comtois, 2012). À cet égard, les configurations de l'espace RhinRhône ressemblent étroitement à cette dernière catégorie si l'on se réfère à son positionnement dans l'itinéraire entre Mer du Nord et Mer Méditerranée. Ce fonctionnement en séquence de corridors se rapproche de la logique de construction des TEN-T, l'espace Rhin-Rhône étant un maillon d'un vaste ensemble à l'échelle continentale, dont l'objectif est de favoriser l'intégration régionale et la coopération économique entre Etats voisins (Arnold, 2005). Ce critère de la morphologie met aussi en évidence le caractère international du corridor puisqu'il est généralement commun à un ou plusieurs pays, avec son pendant politique (Béziat et al., 2014). En découlent des stratégies locales, nationales ou régionales, en Europe notamment avec le réseau TEN$\mathrm{T}$, « dans le cadre de politiques et de pratiques d'aménagement qui tentent de remettre en cause des effets de la fermeture territoriale » (Moutari Elhadji et Giraut, 2013). Dans le cas de l'Union Européenne, ce réseau transnational a pour fonction de dépasser le cadre strict des frontières avec pour finalité l'amélioration des relations de transport à cette échelle.

11 À la lecture des traits essentiels des corridors, l'espace Rhin-Rhône, en tant que maillon dans les échanges à l'échelle européenne, possède de manière intrinsèque les caractéristiques d'un corridor de développement. Dans les discours politiques, la démonstration de son utilité n'est plus à faire, Péter Balázs, coordonnateur pour l'Union Européenne du corridor Mer du Nord-Méditerranée, rappelle à ce propos que la LGV Rhin-Rhône, vaste projet incluant la branche Est, « permettra de réduire un goulet d'étranglement le long de ce corridor multimodal qui s'étend de l'Irlande jusqu'à Marseille $»^{2}$. L'espace Rhin-Rhône, dans cette vision large du corridor constitue donc une partie de l'axe d'intégration logistique permettant de relier un port à son hinterland (Rodrigue, 2007). Toutefois, cette fonction peut être mise en question tant les discours politiques persuasifs peuvent entretenir le mythe du corridor de développement, imaginaire véhiculé par les grands projets de transports réalisés ou projetés qui jalonnent l'histoire de l'espace Rhin-Rhône (Wœssner, 2006). Cette fonction de corridor de développement est d'autant plus incertaine en considérant les activités logistiques qui y sont implantées. À l'exception de Mulhouse à l'extrémité Est, la répartition des EPL (Entrepôts et plateformes logistiques) souligne la faible concentration de celles-ci (Ministère de l'Environnement, de l'énergie et de la mer, 2017).

12 La situation n'est pourtant pas figée. Les perspectives de desserte de la LGV RhinRhône, branche Est comprise, et la création d'un réseau de villes, constituent deux 
modifications majeures dans la trajectoire de cet espace. Comme l'indique C. Comtois : «les corridors peuvent être créés, désaxés ou déclinés avec le temps en fonction de l'introduction de nouvelles technologies, de la construction de nouvelles infrastructures, de l'élaboration de politiques » (Comtois, 2012). Dans ce cadre, LGV et réseau de villes représentent autant de facteurs favorables au développement des activités de transport et logistique au sein de cet espace.

\section{Une politique d'aménagement du territoire favorable au développement du corridor}

\section{La branche Est comme colonne vertébrale de l'espace Rhin-Rhône}

Le contexte favorable des années 1980-1990 pour la réalisation des LGV en France, comme en témoigne le Schéma directeur des LGV de 1991, est à l'origine d'une multitude de projets sur l'ensemble du territoire métropolitain. La LGV Rhin-Rhône en fût l'un d'entre eux. Elle dessine un réseau en " $\mathrm{Y}$ » avec une branche Ouest qui connecte Dijon à la LGV Paris-Lyon dans le nord de la Côte d'Or, et une branche Sud qui relie la capitale bourguignonne à Lyon (illustration 2). Néanmoins, face à l'impératif budgétaire, la réalisation de la branche Est, troisième tronçon du projet, a été préférée avec un phasage en deux temps. Inaugurée en décembre 2011, cette LGV a pour fonction d'améliorer les relations ferroviaires entre Paris et la Suisse, Lyon et Strasbourg, et, à une échelle plus fine, l'accessibilité interurbaine de la plupart des villes, notamment Dijon, Besançon, Montbéliard, Belfort et Mulhouse. Cet équipement s'accompagne de la création de deux gares nouvelles en périphérie des agglomérations de Besançon et de Belfort-Montbéliard, et du raccordement du Canton suisse du Jura via la réouverture de la ligne Belfort-Delle, inaugurée en décembre 2018.

À la différence du Canal à Grand Gabarit Rhin-Rhône, la branche Est est, avec l'autoroute A36, un grand équipement de transport venant légitimer la fonction de corridor de l'espace Rhin-Rhône. Toutefois, ce rôle de maillon pour les circulations nord-sud en Europe peut être mis en cause dans le contexte général de priorisation, voire d'arrêt des projets de LGV en France. Avec l'exemple du rapport de la Commission Mobilité 21 en 2013 (Duron, 2013) et du Conseil d'Orientation des Infrastructures (COI, 2018) publié en début d'année 2018, la priorité est donnée aujourd'hui au réseau de chemin de fer classique et à la rénovation des principaux nœuds ferroviaires français. Concrètement, les perspectives de réalisation du projet dans sa totalité s'éloignent considérablement, y compris la deuxième tranche de la branche Est, puisque les experts n'ont pas préconisé un financement avant 2038. 
Illustration 2 - La desserte de l'espace Rhin-Rhône par la grande vitesse ferroviaire

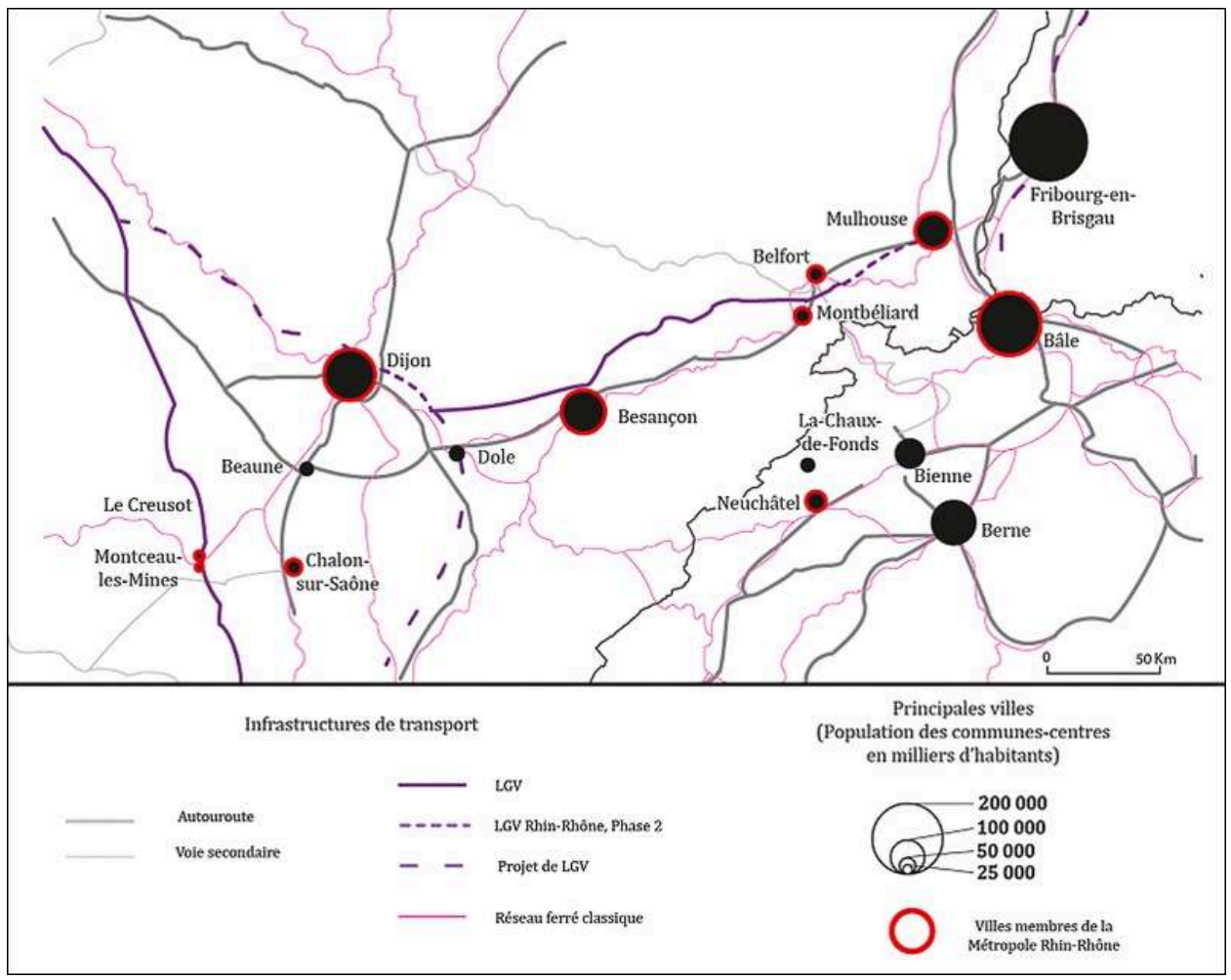

Source : Commission Européenne (TENtec) [site web] https://ec.europa.eu/transport/infrastructure/ tentec/tentec-portal/map/maps.html. Auteur : G. Carrouet, 2019.

Du point de vue de la desserte, la LGV participe à l'amélioration des relations ferroviaires entre les grands foyers urbains (Paris, Lyon, Bâle et Strasbourg) et les agglomérations de taille moyenne de l'espace Rhin-Rhône. Entre 2012 et 2019 d'ailleurs, l'offre de transport en TGV direct est globalement restée stable ${ }^{3}$, quel que soit le type de relation (Tableau 1). Pour les offres Bâle-Paris et Lyon-Strasbourg, le niveau de desserte a peu évolué avec respectivement quatre et cinq TGV directs en 2012 contre cinq et quatre en 2018. Pour le cas des agglomérations appartenant à l'espace RhinRhône, à l'image d'autres réalisations de LGV, la desserte vers Paris est une priorité puisque l'offre est nettement supérieure par rapport aux relations vers Strasbourg et Lyon. La desserte des villes de l'espace Rhin-Rhône est dans l'ensemble restée stable à l'exception de celle de Besançon, ville pour laquelle l'offre a été progressivement déplacée vers la gare TGV périphérique. Cette perte de desserte est par ailleurs partiellement compensée avec une correspondance systématique à Dijon au départ des deux gares de l'agglomération bisontine. Plus globalement, les agglomérations de l'espace Rhin-Rhône bénéficient d'une rente de situation car elles sont positionnées sur les relations entre grands foyers urbains. Par exemple, elles sont situées sur la relation entre Bâle et Marseille qui dessert les gares de l'espace Rhin-Rhône ${ }^{4}$. Sur la même période, le prolongement ou la création de liaisons entre Luxembourg ou Metz et le Sud de la France (Marseille, Montpellier, Nice) a permis d'accroitre la desserte des agglomérations de l'espace Rhin-Rhône. En raison notamment de cette rente, à une échelle plus fine, la LGV a donc engendré un surplus d'accessibilité entre les villes de l'espace Rhin-Rhône. Si le TER est le plus approprié pour les relations de proche en proche, l'infrastructure a engendré une amélioration de la desserte pour les villes les 
plus éloignées. Par exemple, la relation entre Dijon et Mulhouse est passée de $2 \mathrm{~h} 30 \mathrm{à} 1 \mathrm{~h}$ entre 2009 et 2012. Le niveau de desserte a d'ailleurs progressé de trois relations pour atteindre quatorze TGV directs en 2019.

Le cas de l'espace Rhin-Rhône fait figure de particularité dans le modèle à grande vitesse français. Bien que conçue pour répondre aux besoins de transports des grands foyers urbains qui l'entourent, la LGV apporte un surplus d'accessibilité non négligeable aux agglomérations moyennes qui le composent. L'exemple de la relation ferroviaire entre Dijon et Mulhouse souligne particulièrement ces conditions favorables.

Tableau 1 - Nombre de liaisons directes en 2019 et évolution par rapport à 2012

\begin{tabular}{|c|c|c|c|}
\hline & Paris & Lyon & Strasbourg \\
\hline Dijon & $\begin{array}{l}14 \text { TGV }(-2) \\
6 \text { TER }(+2)\end{array}$ & $\begin{array}{l}4 \text { TGV }(-2) \\
16 \text { TER }(-2)\end{array}$ & $4 \mathrm{TGV}(+2)$ \\
\hline Besançon-Viotte & $2 \mathrm{TGV}(-2)$ & $\begin{array}{l}0 \text { TGV }(-1) \\
4 \text { TER }(=)\end{array}$ & 0 TGV (-1) \\
\hline Besançon Franche-Comté TGV & $6 \mathrm{TGV}(-2)^{5}$ & $5 \mathrm{TGV}(+1)$ & $5 \mathrm{TGV}(+1)$ \\
\hline Montbéliard & Aucune desserte & 3 TER $(=)$ & Aucune desserte \\
\hline Belfort & 5 Intercités $(+1)$ & 3 TER $(=)$ & Aucune desserte \\
\hline Belfort-Montbéliard TGV & $8 \mathrm{TGV}(=)$ & $5 \mathrm{TGV}(=)$ & $5 \mathrm{TGV}(=)$ \\
\hline Mulhouse & $9 \mathrm{TGV}(-2)^{6}$ & $5 \mathrm{TGV}(-1)$ & $\begin{array}{l}4 \mathrm{TGV}(-1) \\
34 \mathrm{TER}(+5)\end{array}$ \\
\hline
\end{tabular}

Source : https://www.oui.sncf/billet-train/horaires

\section{Une coopération métropolitaine basée sur la grande vitesse ferroviaire}

17 La mise en service de la branche Est a été perçue par les acteurs locaux comme un levier favorable au développement des coopérations, l'objectif premier étant de favoriser le développement des territoires desservis par cet équipement de transport via la constitution d'un réseau de villes. Celui-ci trouve ses fondements dans les politiques d'aménagement du territoire impactant les villes moyennes dont il est nécessaire de rappeler les principaux traits. À l'échelle française, les premières interventions de l'État en faveur des villes moyennes ont tour à tour favorisé l'implantation des populations et des activités tertiaires et industrielles de 1970 jusqu'aux années 1980. Elles ont également encouragé des concurrences entre ces mêmes villes pour attirer les investissements publics et privés (Santamaria, 2012). Les années 1990 vont marquer un tournant dans ce registre puisque la DATAR va permettre l'émergence de réseaux de villes dont l'objectif, via la mise en commun des moyens et le 
développement d'actions communes, est de mieux résister à la concurrence urbaine des villes voisines. Malgré l'échec de cette première politique en raison du faible résultat qu'elle a engendré (Tesson, 1996), les réseaux de villes vont être de nouveau plébiscités en s'appuyant sur le niveau métropolitain, échelon supérieur de la hiérarchie urbaine. L'objectif est d'affirmer cet échelon sur la scène européenne grâce à des coopérations renforcées entre métropoles de taille moyenne.

De manière générale, les réseaux de villes peuvent se définir comme « des systèmes de relations et de flux à caractère essentiellement horizontal et non hiérarchique, s'établissant entre des centres complémentaires ou semblables " (Camagni et Gibelli, 1994). Cette coopération peut osciller entre une approche globale du développement des villes ou une action centrée sur un équipement commun telle que les infrastructures de transport (Wolff, 2004). Dans le cas de l'espace Rhin-Rhône, les enjeux de développement d'un corridor grâce à ce type de coopérations, s'illustrent à travers les deux réseaux de villes apparus et dont l'essence repose sur la présence d'infrastructures de transport. Dans le premier cas, dès les années 1990, le réseau de villes Rhin-Sud associant les villes de Mulhouse, Belfort, Colmar, Héricourt, Montbéliard et Saint-Louis compte parmi ses principaux objectifs, le renforcement des liaisons avec l'EuroAirport et l'accueil des touristes d'affaires. Disparu dans les années 2000 , ce réseau constitue une première initiative locale à l'origine du pôle de compétitivité « Véhicule du futur » associant le savoir-faire des industries et centres de formation implantés localement.

19 La création d'un réseau de villes élargi à l'ensemble de cet espace dans les années 2000, à la suite d'un appel à projet de la DATAR, représente la deuxième initiative de mise en réseau des villes. L'appel à coopération de 2004 « Pour un rayonnement européen des métropoles françaises $»^{7}$ a pour objectif global d'accroître le rayonnement européen des villes françaises et de favoriser conjointement la croissance des emplois métropolitains supérieurs (EMS), généralement concentrés dans les grandes métropoles de françaises et européennes. Sur les 15 projets retenus figure le « Réseau métropolitain Rhin-Rhône » renommé par la suite "Métropole Rhin-Rhône » qui associe au départ les villes de Dijon, Besançon, Montbéliard, Belfort et Mulhouse. Pour autant, ce réseau a été accepté dans un second temps "en raison de sa faible consistance contrairement aux autres candidatures du Grand Est adossées aux anciennes métropoles d'équilibre, à savoir le Sillon Lorrain (Nancy, Metz, Epinal et Thionville) et l'Eurodisctirct Strasbourg-Ortenau " (Wœssner, 2006). Afin de pallier cette taille critique insuffisante et la dimension européenne qui lui faisait défaut, d'autres entités urbaines ont rejoint le réseau: l'Agglomération trinationale de Bâle (ATB), Neuchâtel pour la Suisse, les régions Bourgogne, Franche-Comté, Alsace et les agglomérations de Chalon-sur-Saône et Le Creusot-Montceau-les-Mines pour la partie française. La gouvernance du réseau repose sur la nomination d'un Président, Jo Spiegel alors maire de Kingersheim et d'un Secrétaire général en relation directe avec les différents services (Développement économique, Transport, etc.) et exécutifs des agglomérations qui le composent.

Outre l'éventail des domaines de coopération (santé, enseignement supérieur/ recherche, culture/loisirs), et la volonté de s'associer dans le secteur qui relève du développement économique ${ }^{8}$, ce réseau de villes fait de la branche Est sa colonne vertébrale avec une véritable doctrine, la nouvelle offre de transport devant permettre «à chaque habitant d'organiser un rendez-vous dans une autre ville de l'axe, aller- 
retour, dans le délai d'une demi-journée » (Métropole Rhin-Rhône, 2008). La création du réseau de villes étendu à la majeure partie des agglomérations de l'espace RhinRhône, témoigne donc des enjeux de développement territoriaux associés aux grands équipements de transport.

\section{Un réseau de villes incarné dans les représentations des acteurs locaux}

21 Les coopérations initiées dans le cadre de la Métropole Rhin-Rhône constituent les prémices, du moins dans les discours, d'une réflexion commune sur l'organisation des activités au sein d'un maillon du couloir de circulation nord/sud en Europe. Dans le cadre d'une thèse de doctorat de 2013 (Carrouet, 2013), il a été question de vérifier l'adéquation entre les discours portés par ce nouveau réseau de villes et les représentations des acteurs locaux sur l'ensemble de l'espace Rhin-Rhône. Après cinq années d'existence, l'hypothèse sous-jacente est de vérifier la présence d'un discours persuasif de la Métropole Rhin-Rhône et son appropriation par les acteurs locaux, dont leurs choix s'orientent en fonction de « leurs propres représentations quant au devenir de l'espace en question » (Gumuchian et Marois, 2000). Sur les 30 entretiens, la majeure partie des interviewés appartient aux services des transports ou du développement économique des agglomérations (Dijon, Dole, Vesoul, Besançon, Montbéliard, Mulhouse), du Conseil départemental (Jura et Haute-Saône) et du Conseil de régional (Franche-Comté), auxquels s'ajoute un représentant Suisse (Canton du Jura). Des organismes consulaires (CCI du Doubs et du Territoire de Belfort), des représentants des agences d'urbanisme (Belfort et Montbéliard) complètent le panel. Enfin, pour ne pas ignorer le rôle des transports dans l'émergence de ces coopérations, le choix a été d'interviewer des acteurs du secteur des transports (SNCF mission Rhin-Rhône, SNCF Réseau ; OFT), la FNAUT et un représentant de l'association de lobbying Trans Europe TGV Rhin-Rhône Méditerranée.

Dans le cadre des entretiens semi-directifs menés de janvier à juillet 2011, les individus interrogés étaient invités à donner leur représentation de "l'espace Rhin-Rhône » en mobilisant des critères géographiques (situation) ou matériels (importance des circulations, répartition des activités économiques) sans faire mention de la Métropole Rhin-Rhône, son apparition spontanée dans les discours des individus interrogés étant, de fait, une preuve de son existence. A minima, cela peut souligner le caractère performatif de la communication de cette entité qui se traduisant dans les propos des interviewés.

23 Ces entretiens ont fourni deux résultats essentiels. D'une part, l'espace Rhin-Rhône se définit, selon les individus interrogés et de manière unanime, par le critère du transport, puisqu'il est couloir " naturel », « de transit » dans les échanges entre Nord et Sud de l'Europe. D'autre part, le deuxième résultat marquant provient de la discordance dans les représentations au sujet de sa dimension territoriale. Si pour près de la moitié des acteurs (17 sur 30), cette portion d'espace se définit uniquement par la dimension citée précédemment, pour la seconde moitié, l'arrivée de la grande vitesse ferroviaire et la création du réseau de villes sont perçues comme deux facteurs clés d'une dimension territoriale représentée par la Métropole Rhin-Rhône, première réalité qui dépasse les découpages institutionnels classiques. Cette réalité est d'autant plus vraie pour les représentants des agglomérations membres qui partagent quasi 
systématiquement ce point de vue. S'il est difficile d'expliquer précisément pour quelles raisons cette dimension territoriale est davantage présente chez ce type d'acteurs, il est certain que la communication de ce réseau de villes a été particulièrement efficace auprès des services et du grand public à travers l'organisation d'évènements culturels (exposition d'œuvres d'art « Utopies et innovations entre Rhin et Rhône ») ou de sponsoring (Festival de musiques actuelles "TGV Generiq »), bien qu'il n'y ait pas, à cette même période, de coopérations avancées sur des thématiques relevant du transport et du développement économique, secteur pourtant identifiés comme décisifs.

Les discours portés par la Métropole Rhin-Rhône ont particulièrement relevé d'un caractère performatif. La forte présence médiatique et l'engouement manifeste autour de la branche Est, malgré les difficultés d'initier des coopérations interrégionales et transnationales, ont permis à cette entité de gagner en légitimité. Au-delà des représentations des acteurs, l'analyse des stratégies collectives en matière de gestion du tracé de la LGV et des programmes de développement des abords des gares permettent d'identifier la capacité des acteurs à coopérer et sous quelles conditions.

\section{Appropriation collective de la grande vitesse et développement territorial}

Au cœur de la question portant sur les coopérations entre villes de l'espace Rhin-Rhône figure le critère de l'appropriation de ce couloir de circulation et de ses infrastructures, et de tous les éléments auxquels il renvoie, dans le champ plus vaste des relations entre transports et territoires. L'appropriation peut se définir comme "l'adaptation de quelque chose à un usage défini ou à une destination précise » (Segaud et al., 2003), où l'adaptation «traduit un objectif d'harmonie entre une chose et l'usage auquel on la destine, un heureux appariement entre deux objets, deux actions ou entre un sujet et un objet " (Segaud et al., 2003). Dans le cas présent, le couloir de circulation et les grands équipements de transport sont perçus comme des leviers favorables à l'amélioration de l'accessibilité et au développement territorial. Il est donc question d'analyser successivement l'appropriation des acteurs de la LGV et les transformations territoriales projetées sur leur territoire, dans le cas de la définition du tracé d'une part, et les stratégies de valorisation des abords des gares desservies par la grande vitesse ferroviaire d'autre part.

\section{Une appropriation collective à l'origine du tracé de la LGV}

La première appropriation questionne l'émergence de coopérations plusieurs années avant la création du réseau de villes. Souvent identifiées à la quête du Graal, les LGV peuvent laisser place, très tôt dans l'historique des projets de desserte, à des coopérations pour défendre le projet sur les scènes nationale et européenne.

Dans l'historique du projet, le premier fait marquant de la LGV Rhin-Rhône remonte au début des années 1980 dans un contexte de multiplication des initiatives en France métropolitaine et de la mise en service du premier tronçon entre Paris et Lyon. Les élus de la partie nord de la région Franche-Comté et du sud de l'Alsace œuvrent pour la réalisation d'une LGV desservant cette portion de territoire, en réaction à son évitement manifeste dans les premières esquisses du projet de ce qui deviendra la LGV 
Est Européenne. La proposition consiste en un prolongement de la LGV Sud-Est pour rejoindre Belfort, Mulhouse et la partie nord de la Suisse grâce à l'électrification de la ligne classique Vesoul-Belfort. L'association «Trans Europe Londres-Paris-Aisy-BâleAdriatique » est créée en 1986 pour fédérer les acteurs locaux autour de ce projet. Son principal représentant et instigateur est Jean-Pierre Chevènement dont les fonctions ministérielles successives dans la décennie 1980 auront un impact sur le devenir du projet de desserte à grande vitesse.

Non retenu en raison de son coût, la proposition s'efface au profit d'un nouveau projet dont le succès va reposer sur des gains d'accessibilité ferroviaires pour l'ensemble des villes de l'espace Rhin-Rhône. Cette initiative, dont la région Franche-Comté est le premier porteur, prévoit une amélioration substantielle de la desserte des villes de Dijon, Dole et Besançon situées à l'ouest, non concernées par le tracé précédent. La desserte proposée va donner naissance à une coalition de projet, c'est-à-dire "des organisations constituées principalement d'élus et d'acteurs socioéconomiques pour promouvoir des projets particuliers ou le réseau dans son ensemble » (Blatrix, 2002). La coalition de projet, signe d'une appropriation collective de la grande vitesse ferroviaire, s'incarne par l'adhésion de la majorité des acteurs de l'espace Rhin-Rhône à l'association de lobbying qui devient la cheville ouvrière dans la défense de la LGV Rhin-Rhône.

$\mathrm{Au}$ début de la décennie 1990, le travail de fond mené par cette association dont l'appellation est devenue "Trans Europe TGV Rhin-Rhône Méditerranée ", évoquant par ailleurs la fonction de maillon que représente l'espace Rhin-Rhône, va permettre de franchir les différentes étapes nécessaires à sa réalisation. La LGV Rhin-Rhône, composée de ses trois branches, sera notamment inscrite au schéma directeur européen en décembre de la même année et à son équivalent en France en avril 1992. Le caractère politique de ce schéma (Zembri, 1997) au regard des nombreux projets retenus dans un contexte où les élus ont exprimé le souhait de projeté une LGV sur leur territoire, l'a rendu irréalisable. Le projet de LGV Rhin-Rhône, pour cette raison, a été segmenté puisque seule la première phase de la branche Est a été réalisée à ce jour.

Initiée au début des années 1980, l'initiative locale de raccordement au réseau à grande vitesse s'est peu à peu transformée en quête collective de l'ensemble des acteurs de l'espace Rhin-Rhône avec le travail de l'association de lobbying. Sans que cela ne soit paradoxal, la concertation sur le schéma de desserte menée par deux médiateurs mandatés par l'État entre 2008 et 2010, réunissant RFF, SNCF et les collectivités territoriales ${ }^{9}$ met en évidence l'effacement de la coalition de projet au profit de l'intérêt porté par chaque collectivité à la desserte de son propre territoire. Bien que présente lors des rendez-vous collectifs, l'association n'a pas pesé dans les négociations, l'objectif étant de défendre avant tout la réalisation de la deuxième tranche de la branche Est selon Claude Liebermann, l'un des deux médiateurs ${ }^{10}$. Le symptôme marquant davantage l'affaiblissement de cette coalition et la faible consistance du réseau de ville, est l'absence de la Métropole Rhin-Rhône puisqu'elle n'a pas pesée dans les négociations. Le médiateur souligne à ce propos «qu'il n'y a pas de volonté collective par rapport à la Métropole Rhin-Rhône, celle-ci étant plus un concept théorique que pratique». 


\section{Des projets de développement redondants à proximité des gares}

31 Les stratégies de développement locales aux abords des gares en lien avec la mise en service de la LGV offrent une autre perspective en matière d'appropriation collective et de coopération à l'échelle de l'espace Rhin-Rhône. En conformité avec les discours de la Métropole Rhin-Rhône, les coopérations dans le cadre de la gestion du tracé offrent des perspectives pour un développement concerté des zones d'activités à proximité des gares.

D'un point de vue scientifique, cette entrée s'insère dans l'analyse des effets engendrés par la nouvelle offre de transport. Ce champ de recherche trouve ses origines dans la persistance d'un schéma de pensée chez les décideurs locaux qui voient dans l'infrastructure et les gains d'accessibilité associés, un facteur de développement automatique des territoires. Sans revenir en détail sur les nombreux travaux consacrés à la question des effets structurants (Bérion et al., 2007 ; Plassard, 2003 ; FacchinettiMannone, 2003), la plupart d'entre eux soulignent la combinaison de facteurs nécessaires à l'apparition des effets supposés et l'adaptation réciproque entre infrastructure de transport et territoire, les autoroutes ou LGV étant généralement un facteur de renforcement des tendances préexistantes.

La méthodologie repose sur un travail de recensement des projets de développement mené entre décembre 2011 et janvier 2012 réactualisé en novembre 2018, soit 8 années plus tard. Pour vérifier l'existence d'une gestion concertée des programmes de développement aux abords des gares, seuls les projets initiés à proximité des principales agglomérations de l'espace Rhin-Rhône (Dijon, Besançon, Belfort, Montbéliard et Mulhouse) sont retenus.

Les résultats sont sans appel. La nouvelle offre de transport est un facteur favorable à l'émergence de projets de développement territoriaux, les acteurs de la plupart des collectivités territoriales ayant anticipé très tôt l'arrivée de la nouvelle desserte. Toutefois, cette appropriation ne relève pas d'une stratégie globale partagée entre l'ensemble des territoires, comme en témoigne l'absence de gouvernance pour des agglomérations seulement distantes de 20 à 30 minutes en TGV.

Si à Dijon aucun projet d'ampleur n'est apparu sur la période en raison des configurations du quartier de gare dont les emprises foncières sont restreintes et du caractère ancien de la desserte TGV, la ville étant raccordée au réseau depuis les débuts des années 1980, les stratégies développées dans les trois autres agglomérations soulignent l'absence d'unité à l'échelle de cet espace. À Besançon, le partage de la desserte entre la gare Besançon Franche-Comté TGV qui capte l'essentiel de l'offre grande ligne et la gare Besançon Viotte, induit la coexistence de projets distincts à proximité des deux points de desserte. Au centre-ville, un projet d'éco-quartier sur d'anciennes friches ferroviaires est projeté peu de temps avant la mise en service de la LGV mais repoussé à des échéances ultérieures, la première pierre ayant été posée en juin 2018. Dans sa globalité, le projet prévoit la réalisation sur $44000 \mathrm{~m}^{2}$ d'un programme de création de logements, de commerces et services et de deux bâtiments nommés « Signal » dont l'un a pour vocation d'accueillir des fonctionnaires d'État et de la région Franche-Comté. Dans le périmètre de la Communauté d'agglomération du Grand Besançon, un centre d'affaires nommé «Le Signal » a été inauguré en septembre 2017 à proximité de la gare TGV. À l'image des agglomérations précédemment desservies par d'autres LGV, cet équipement a pour fonction d'héberger des entreprises 
de services résidentes et propose de la location de bureaux ou de coworking à la demijournée ou journée.

Dans le nord de l'ancienne région Franche-Comté, les stratégies se résument essentiellement par le projet de ZAC «La Jonxion » initiée dès 2010 à proximité de la gare Belfort Montbéliard TGV, sur une surface de 150 hectares. Dans une logique similaire à l'agglomération bisontine, la Jonxion 1, dont la commercialisation a commencé plus tôt, accueille des entreprises dans le secteur du tertiaire supérieur, un hôtel et un centre d'affaires "AZAP » destinés à l'accueil temporaire d'activités tertiaires, basé sur un modèle similaire à celui du bâtiment «Le Signal » à Besançon. Devant un taux de remplissage rapide de la première phase, la "Jonxion Core", deuxième zone prévue à proximité immédiate sera également dédiée au secteur tertiaire.

Les stratégies de développement locales à proximité de la gare centrale de Mulhouse sont semblables aux deux agglomérations franc-comtoises puisque leur contenu est avant tout orienté sur le développement des activités tertiaires. Engagé dès 2011, le projet de l'agglomération prévoit la réalisation de près de $57000 \mathrm{~m}^{2}$ de bureaux. À l'heure actuelle, 4 immeubles sont déjà construits. Trois d'entre eux accueillent, sur tout ou partie de la surface, des sièges d'entreprises (Banque Populaire Alsace Lorraine Champagne Ardenne, Macif par exemple), le dernier réservé à l'activité hôtelière (Ibis). À terme, d'autres bâtiments destinés aux activités tertiaires et de séminaires, à l'image des projets franc-comtois, viendront compléter ce programme.

Les stratégies de développement locales à proximité des points de desserte soulignent l'absence d'une gouvernance partagée, et donc d'un espace de projet à l'échelle de la Métropole Rhin-Rhône qui appelait de ses vœux ces coopérations multiples. La redondance des projets est le symptôme des rivalités entre collectivités territoriales. La disparition de la Métropole Rhin-Rhône en 2012, symbole de cette appropriation collective de la grande vitesse, témoigne de l'incapacité des acteurs à développer des programmes de développement communs sur une portion d'espace composée de villes moyennes au rayonnement nécessairement limité par rapport aux grandes métropoles voisines.

\section{Conclusion}

39 L'espace Rhin-Rhône, dans son actualité la plus récente, souligne les enjeux de développement d'un maillon du plus vaste corridor Mer du Nord - Méditerranée dont la dimension politique et l'objectif d'intégration régional n'est plus à démontrer.

La grande vitesse ferroviaire matérialisée par la branche Est de la LGV Rhin-Rhône a été perçue comme un facteur favorable à l'émergence de coopérations au sein de cet espace multipolaire, et d'un possible agencement des activités en son sein. Cette appropriation collective de la grande vitesse ferroviaire a existé sur les 3 décennies qui jalonnent l'histoire du projet de LGV, à travers la coalition de projets pour la définition du tracé et la création du réseau de villes de la Métropole Rhin-Rhône.

Pourtant, ce cas d'étude situé à la croisée des problématiques des interrelations entre transports et territoires et des effets des politiques d'aménagement du territoire favorables aux villes moyennes, met en évidence les inerties territoriales venant contrarier les perspectives de développement des corridors. Si la LGV représente un 
facteur favorable pour les coopérations entre décideurs locaux, celles-ci se sont produites dans une logique de guichet, l'adhésion collective au projet de desserte s'apparente à un compromis nécessaire pour la quête de la grande vitesse ferroviaire. Les jeux d'acteurs quasi institutionnalisés dans les années 1980-1990 se sont effacés progressivement au profit des velléités de développement local de chaque agglomération comme en témoigne la redondance des projets à proximité des points de desserte des agglomérations de Besançon et de Belfort-Montbéliard seulement distantes d'une centaine de kilomètres.

L'espace Rhin-Rhône ne peut prétendre au statut de corridor de développement en raison de l'absence d'une gouvernance sur le plan régional et de la présence de maillons concurrents à l'échelle européenne, dont le corridor Rhin-Alpin, qui viennent contrarier ce statut d'interface. L'absence de discours et de mesures en faveur du développement $d u$ fret ferroviaire et de la fixation des activités logistiques renforcent ce constat. Ce cas d'étude et cette perspective de développement contrariée qu'il soulève ne sont pas moins enrichissants lorsqu'il est question du devenir et des trajectoires de certains espaces, à l'image des corridors, dont la dimension circulatoire est marquée. Dépourvu de dimension territoriale, l'espace Rhin-Rhône a été un temps un espace de projet, c'est-à-dire un "espace social, plus ou moins territorialisé, qui s'organise autour de rivalités, un champ au sein duquel se construit un problème à résoudre » (Nahrath et Varone, 2007) : celui de la desserte de villes moyennes par la grande vitesse ferroviaire et des perspectives sous-jacentes de développement territorial. Dans le cas de l'espace Rhin-Rhône, l'infrastructure de transport a été davantage un facteur de renforcement des tendances préexistantes en matière de développement territorial au regard des rivalités existantes entre les zones d'aménagement initiées à proximité des points de desserte.

\section{BIBLIOGRAPHIE}

Arnold J., 2005. Best Practices in Corridor Management. The World Bank, 45 p.

Bérion P., Joignaux G., Langumier J-F., 2007. L'évaluation socio-économique des infrastructures de transport : Enrichir les approches du développement territorial. Revue d'Économie Régionale et Urbaine, $\mathrm{n}^{\circ}$ 4, p. 651-676.

Béziat A., Debrie J., Heitz A., 2014. L'aménagement des corridors de transport : outil d'action publique? Analyse comparative de l'Axe-Seine (France) et du corridor Ontario-Québec (Canada). Géotransports, $\mathrm{n}^{\circ}$ 4, p. 85-98.

Blatrix C., 2002. Devoir débattre. Les effets de l'institutionnalisation de la participation sur les formes de l'action collective. Politix, vol. 15, n 57. p. 79-102.

Camagni R., Gibelli M C., 1994. Réseaux de villes et politiques urbaines. Flux, n 16, p. 5-22

Carrouet G., 2013. Du TGV Rhin-Rhône au «territoire » Rhin-Rhône : réticularité, mobilité et territorialité dans un espace intermédiaire. Thèse de Doctorat en Géographie, Université de Bourgogne, 526 p. 
Comtois C., 2012. Définition et périmètre des grands corridors de transport fluvio-maritime, In Alix Y., Les corridors de transport. Cormelles-le-Royal, Editions Ems, p. 65-87.

COI (Conseil d'orientation des infrastructures de transport), 2018. Mobilités du quotidien : répondre aux urgences et préparer l'avenir. La Documentation Française. 19 p.

Debrie J, Comtois C., 2010. Une relecture du concept de corridors de transport : illustration comparée Europe/Amérique du Nord. Les cahiers scientifiques du transport, n 58, p. 127-144.

De Ruffray S., 2004. Le Grand Est : un espace différencié, interface marginale aux portes de l'Europe. Revue Géographique de l'Est, vol. 44, n³-4, p. 97-106.

Dézert B., 1969. La Croissance industrielle et urbaine de la Porte d'Alsace : essai géographique sur la formation d'un espace régional en fonction de l'attraction industrielle. Paris, Société d'édition d'enseignement supérieur, $520 \mathrm{p}$.

Dézert B., 2003. La mutation des moyens d'échange, In Auphan É., Dézert B., L'Europe en mouvement : population, transports, aménagement, tourisme. Paris, Ellipses, $319 \mathrm{p}$.

Duron P., 2013. Mobilité 21 - « Pour un schéma national de mobilité durable ». La Documentation Française. $91 \mathrm{p}$.

Facchinetti-Mannoce V., 2003. Les gares TGV périphériques : de nouveaux pôles de développement? In Siino C., Laumière F., Leriche F., Métropolisation et grands équipements structurants. Toulouse, Presses Universitaires du Mirail, p. 71-84.

Gibert A., 1930. La Porte de Bourgogne et d'Alsace, Trouée de Belfort : étude géographique. Paris, Armand Colin, $632 \mathrm{p}$.

Gumuchian H., Marois C., 2000. Initiation à la recherche géographique. Montréal, Presses de l’Université de Montréal, 425 p.

Métropole Rhin-Rhône, 2008. Entre Rhin et Rhône une métropole d'un nouveau type. 56 p.

Ministère de l'Environnement, de l'énergie et de la mer, 2017. Atlas des entrepôts et des aires logistiques en France en 2015. 112 p.

Moutari Elhadji M, Giraut F., 2013. Le corridor de transhumance au Sahel : un archétype de territoire multisitué ? L'Espace géographique, vol. 42, n 4, p. 306-323.

Nahrath S., Varone F., 2007, Les espaces fonctionnels comme changements d'échelles de l'action publique. In Faure A., Leresche J-P., Muller P., Nahrath S., Action publique et changements d'échelles : les nouvelles focales du politique. Paris, L'Harmattan, p. 235-249.

Ollivro J., 2000. L'homme à toute vitesse : de la lenteur homogène à la rapidité différenciée. Rennes, Presses universitaires de Rennes, $179 \mathrm{p}$.

Plassard, F., 2003. Transport et territoire. Paris, La Documentation française, $104 \mathrm{p}$.

Priemus H., Zonneveld W., 2003. What are corridors and what are the issues? Introduction to special issue: The governance of corridors. Journal of Transport Geography, vol. 11, p. 167-177.

Richer C., Bérion P., 2010. Le rôle des grandes infrastructures dans la structuration des espaces régionaux : le cas de l'arrivée du TGV dans le réseau métropolitain Rhin-Rhône. BELGEO, vol. 1-2, p. $160-169$.

Ripoll F., Veschambre V., 2005. Introduction. L'appropriation de l'espace comme problématique. Norois [En ligne], n 195, p. 7-15. https://journals.openedition.org/norois/477 
Rodrigue J-P., 2007. Gateways, corridors and global freight distribution: transpacific issues. 1st International Conference on Gateways and Corridors [En ligne], 24 p. http://www.gatewaycorridor.com/reginaroundtable/documents/papers/JPRPaperGatewaysCorridorsRegina.pdf

SANTAMARIA F., 2012. Les villes moyennes françaises et leur rôle en matière d'aménagement du territoire : vers de nouvelles perspectives ? Norois [En ligne], n²23, p. 13-30. https:// journals.openedition.org/norois/4180

Segaud M., Brun J., Driant J-C., 2003. Dictionnaire critique de l'habitat et du logement. Paris, Armand Colin, $451 \mathrm{p}$.

Tesson F., 1996. Les réseaux de villes en France, recherche sur le rapport de l'élu local à l'espace. Thèse de doctorat de Géographie, Université de Pau et des Pays de l'Adour, 267 p.

Wœssner R., 1996. Mythe et réalité de l'espace Rhin-Rhône : la dynamique industrielle comme facteur de recomposition territoriale. Thèse de Doctorat de Géographie, Université de Franche-Comté, 531 p.

WÆSSNER, R., 2006. L'éternel retour du Corridor Rhin-Rhône. Annales de Géographie, vol. 1, n 647, p. 2-25.

Wolff, J-P., 2004. Réseaux de villes et réseaux culturels dans l'Ouest. Norois [En ligne], nº 190, p. 97-109. https://journals.openedition.org/norois/81

Zembri P., 1997. Les fondements de la remise en cause du Schéma Directeur des liaisons ferroviaires à grande vitesse : des faiblesses avant tout structurelles. Annales de géographie, $n^{\circ}$ 593-594, p. 183-194.

\section{NOTES}

1. Cette délimitation a été proposée dans le cadre d'un travail de doctorat soutenu en 2013.

2. Communiqué de presse du 16 mars 2017 de l'Association Trans Europe TGV Rhin-Rhône Méditerranée [En ligne]. http://www.transeuropetgv.net/nos-actions/communiques-de-presse/ communique-de-presse-de-lassociation-du-16-mars-2017/

3. Relevé des dessertes réalisé sur un jour ouvrable du mois de juin 2012 et juin 2019 à partir du site SNCF.

4. Cette liaison est supprimée en 2019 en raison de la baisse globale de l'offre de TGV passant par la gare de Lyon Part-Dieu en raison des travaux d'agrandissement de capacité.

5. Pour la direction parisienne, les usagers au départ de Besançon ont davantage de correspondances à Dijon, prochaine gare desservie dans le sens Province-Paris.

6. La perte de desserte de deux TGV est compensée par la mise en service du second tronçon de la LGV Est Européenne. Le différentiel de temps de trajet est de 9 minutes en considérant les meilleurs temps de parcours ( $2 \mathrm{~h} 40$ pour le trajet via LGV Rhin-Rhône, $2 \mathrm{~h} 49$ pour le trajet via LGV Est Européenne).

7. DATAR, 2004. Pour un rayonnement européen des métropoles françaises. Appel à coopération métropolitaine. $16 \mathrm{p}$.

8. Le document de référence de la Métropole Rhin-Rhône signale à ce propos : «c'est en luimême que ce territoire entre Rhône et Rhin trouve les raisons et la force d'organiser sa cohésion propre, en s'appuyant sur un socle industriel puissant, sur ses nombreux savoir-faire reconnus à l'image de ses pôles de compétitivité, de ses systèmes productifs locaux et de ses entreprises de renom » (Métropole Rhin-Rhône, 2008).

9. Le financement des collectivités s'élève à $28 \%$ du coût total de la LGV.

10. Le médiateur a été rencontré lors du travail de doctorat. 


\section{RÉSUMÉS}

L'espace Rhin-Rhône, comprenant un ensemble d'agglomérations de Dijon à Bâle, illustre les enjeux de développement d'un espace inclus dans le corridor Mer du Nord - Méditerranée. L'arrivée de la grande vitesse ferroviaire a été perçue par les acteurs locaux comme un facteur favorable à l'émergence de coopérations à travers la constitution d'un réseau de villes, la Métropole Rhin-Rhône. Sous impulsion de l'État, cette initiative représente un enjeu pour un espace dont la dimension circulatoire est certaine. Pourtant, après 8 années de service de la LGV, le bilan est décevant au regard des ambitions de départ. Si les enjeux d'accessibilité ont fédéré les acteurs locaux, le développement territorial souhaité à proximité de chaque gare a affaibli cette perspective de coopération. En témoigne, la redondance des zones d'activités à vocation tertiaire crées dans des agglomérations moyennes, séparées seulement d'une centaine de kilomètres.

The Rhin-Rhône space that includes a collection of agglomerations from Dijon to Bâle, illustrates the stakes of the development of a space enclosed in the Mediterranean Sea-Northern Sea corridor. The arrival of high speed train lines was perceived by the local stakeholders as a favorable contributor to the birth of cooperations through the constitution of a network of cities: the Rhin-Rhône Metropolis. Under the impulsion of the state, this initiative represents a stake for a space whose circulatory dimension is certain. However, after eight years of high speed train service, the results are disappointing considering the initial ambitions. The accessibility stakes may have federated the local stakeholders, but the desired territorial development close to each train station has weakened this cooperation perspectice. Reflective of that: the redundancy of zones of activity in tertiary focus resulted in medium agglomerationes, only separated by around 100 kilometers.

\section{INDEX}

Mots-clés : LGV, corridor, réseau de villes, appropriation, effets structurants

Keywords : High speed rail, corridor, network of cities, appropriation, structuring effects Thèmes : Sur le Champ - Sur le Terrain

\section{AUTEUR}

\section{GUILLAUME CARROUET}

Guillaume Carrouet, guillaume.carrouet@univ-perp.fr, est maître de conférences à l'Université de Perpignan, IUT de Perpignan, UMR ART-Dev 5281. Il a récemment publié :

- Carrouet G., 2018. Dégradation de la desserte ferroviaire interurbaine et périphérisation du département des Pyrénées-Orientales. Les cahiers scientifiques du transport, $\mathrm{n}^{\circ}$ 74, p. 3-28.

- Facchinetti-Mannone V., Carrouet G., Bérion P., 2016. Potentiel intermodal et pratique de rabattement des voyageurs des gares ex-urbanisées. In Chapelon L., Transports et intermodalité.

ISTE éditions, p. 237-260.

- Carrouet G., Bérion P., Facchinetti-Mannone V., Richer C., 2013. Les stratégies d'anticipation des « effets » territoriaux des grands équipements de transport : le cas du TGV Rhin-Rhône.

Géotransports [En ligne], n¹-2, p. 35-50. http://geotransports.fr/Revue/pp.

51_66_V.Facchinetti_Mannone_Gares_TGV_peripheriques.pdf 